\title{
Letter to the Editor Open Journal of Applied Biosensor: Point-of-Care Biosensing and Environment Monitoring
}

\author{
Ajeet Kaushik \\ BioMEMS and Microsystems Laboratory, Department of Electrical and Computer Engineering, \\ Florida International University, Miami, USA \\ Email: ajeet.npl@gmail.com
}

Received June 25, 2013; revised July 30, 2013; accepted August 12, 2013

Copyright (C) 2013 Ajeet Kaushik. This is an open access article distributed under the Creative Commons Attribution License, which permits unrestricted use, distribution, and reproduction in any medium, provided the original work is properly cited.

\section{Dear Editor,}

Open journal of applied biosensor (OJAB) is a multidisciplinary journal devoted to research, design development and integration of biosensors and bioelectronics for point-of-care (POC) biosensing and environmental monitoring. The goal of this journal is to provide an advanced platform to motivate researchers to explore enabling new technologies for design and development of biomaterials, biosensor, bioelectronics including, biological materials, nanobiosensors, instrumentation, signal treatment and uncertainty estimation in biosensors, new biosensor fabrication strategy, biosensor interfaces and membrane technology, DNA chips, cell/tumor trapping for diagnostics, lab-on-a-chip technology, biosensor integrated with microfluidic devices for POC.

Biosensors are integrated miniaturized analytical devices that employ biological material (enzymes, antibodies, nucleic acids, tissue, microorganisms, organelles, cell receptors), a biologically derived material (recombinant antibodies, engineered proteins, aptamers) or a biomimic (synthetic receptors, biomimetic catalysts, combinatorial ligands, imprinted polymers etc.) as the sensing element immobilized matrix attached transducer microsystem (optical, electrochemical, thermometric, piezoelectric, magnetic or micromechanical). For examples, DNA biosensor, aptamers based biosensor, immunosensors, enzymebased biosensors, and organism/whole cell-based biosensors. They have the exquisite selectivity of the biomolecule and the processing power of the modern microelectronics and optoelectronics to offer powerful new analytical tools with applications in medical diagnostics. Wherein, physiochemical change is produced by specific interactions between the target analyte and bio-recognition element that is detected in a transducer. Due to their specificity, speed, portability and low cost, biosen- sors offer exciting opportunities for numerous applications in medicine, biomedical research, drug discovery, environmental sensing, food quality and processing, process industries, forensic and homeland security. Exploring new nanostructured immobilizing platforms for signal amplification, understanding of bio-material interfacial properties to improve the analytical performance and robustness are within the scope of OJAB.

The emerging fields of biosensor and bioelectronics seek to exploit biology in conjunction with nanomaterials and their integration with MEMS in a wider context, encompassing for POC and environmental sensing. Systems for monitoring physiological variables on a daily basis at POC are deemed as the cornerstone technology for improved health care delivery at reduced costs. Pointof-care sensors that can quantify critical physiological parameters as a function of one's surroundings can provide data enabling better diagnosis and better treatment strategies. MEMS systems have enabled the development of POC chemical and biological assays. The high degree of automation accuracy and precision that exists in systems also eliminates error often associated with human handling and thereby reduces the percentage of false positive results. Thus focus has shifted towards integrating biosensors into a microfluidic environment with a goal to create POC sensors.

The increasing number of potentially harmful pollutants in the environment has resulted in stringent legislation toward environmental regulation. There is a critical need to monitor contaminants and toxins in air (volatile compounds and gases), water (heavy metals, virus and bacteria), soil (chemicals, minerals) and food (bacteria, viruses, toxins, mycotoxins, pyrethroids, etc.). Where online and continuous monitoring of environmental variable is imperative, traditional laboratory-based analytical 
methods will not suffice. POC systems driven by biosensor integrated microfluidic technology is a key player in the realization of such environmental sensors.

For the success and to maintain consistency within the scope of the OJAB, the journal will accept reviews, research papers, short communication and technical notes which describe enabling technologies, new sensing strategies, new diagnostic tool, bio-chips, miniaturized biomedical devices with new concepts and understanding of the research field with the real application and commercial prospects. 